\title{
COMPARISON OF PRE- AND POST-IMPLANTATION MECHANICAL BEHAVIOR OF COMPOSITE HERNIA MESHES
}

\author{
Miglena Doneva \\ Medical University-Sofia, Faculty of Pharmacy, Sofia, Bulgaria \\ Bulgarian Academy of Sciences, Institute of Mechanics, Sofia, Bulgaria \\ e-mail: miglena_doneva@abv.bg \\ Dessislava Pashkouleva \\ Bulgarian Academy of Sciences, Institute of Mechanics, Sofia, Bulgaria \\ e-mail:dessip@imbm.bas.bg \\ STOYAN SOPOTENSKI \\ Hospital "Saint Sofia", Sofia, Bulgaria \\ e-mail: sopotensky@abv.bg \\ Guenka Petrova \\ Medical University-Sofia, Faculty of Pharmacy, Sofia, Bulgaria \\ e-mail: guenka.petrova@gmail.com
}

\begin{abstract}
Implantation of synthetic meshes provokes complications which require long-lasting follow-up. The mesh materials exposed to physiological conditions demonstrate differences in failure load, stiffness and extension. Mechanical alterations of explants made of polyester were investigated using a uni-axial tensile test and a relaxation test. The tensile strength of the explanted meshes changed in the transversal direction. The elastic modulus in the same direction increased two times. A magnified mesh image displayed mesh alteration. The relaxation tests revealed that the initial stress decreases after implantation while the reduction of stress increases in the interval 50\%-96.6\%.
\end{abstract}

Keywords: biomechanics, explanted surgical mesh, elastic and viscoelastic properties

\section{Introduction}

The use of meshes in hernia repair is considered the most effective approach nowadays. The implanted meshes are synthetic (non-absorbable prosthesis), composite (a combination of absorbable and non-absorbable fibres) and coated with an absorbable or non-absorbable barrier.

Usually hernia operation with a mesh results in lower recurrence rates but it can provoke also serious adverse effects such as infection, pain and local rigidity of the abdominal wall. In order to prevent foreign body sensation or discomfort because of wall stiffness, the elasticity of the abdominal wall is assessed in vivo or in vitro. (Costello et al., 2007; Klosterhalfen et al., 2005; Song et al., 2006; Tran et al., 2016; Wood et al., 2013). Some authors have focused on the in vivo characterization of the mechanical response of the human abdominal wall using ultrasound shear wave elastography, insufflation of the peritoneal cavity and ultrasound scanning or an indentation device (Song et al., 2006; Tran et al., 2016). Decreased abdominal wall elasticity is measured by decreased elongation and increased stiffness of the complex tissue-mesh.

In vitro characterization of the mesh-tissue composite is based on the investigation of explanted meshes. The mutual influence of the mesh material and tissue is investigated in animal and human studies. 
The adhesion formation and shrinkage of Parietex were investigated in animal studies from Bellón et al. (2005), Jacob et al. (2007), Judge et al. (2007), Kayaoglu et al. (2005) and McGinty et al. (2005). Jacob et al. (2007) and Judge et al. (2007) reported shrinkage of Parietex. Jacob et al. (2007) reported that the Parietex Composite (PCO) mesh showed less shrinkage in comparison to Proceed (34\% vs. 29.7\%) and more shrinkage than polypropylene (PP) mesh $(29.7 \%$ vs. 25.4\%). Judge et al. (2007) also reported that the PCO mesh was superior in terms of adhesion prevention, but underwent considerable shrinkage in the experimental model. The PCO mesh underwent more shrinkage than the coated PP mesh (Sepramesh) at both 1 month $(38.2 \%$ vs. $18.1 \%)$ and 5 months (17.4\% vs. $6.1 \%)$. The main conclusion was that the PCO mesh was superior in terms of adhesion prevention, but showed considerable shrinkage.

Other animal studies documented the antiadhesive properties of the PCO mesh. McGinty et al. (2005) studied adhesion formation and abdominal wall ingrowth after laparoscopic ventral hernia repair in a porcine model. PP, expanded polytetrafluoroethylene (ePTFE), and PCO meshes were implanted and after 28-day adhesion formation, and the fibrous ingrowth and shrinkage among the types of mesh were compared. The main conclusion stated that the PCO mesh had fewer and less severe adhesions than ePTFE or PP mesh.

Kayaoglu et al. (2005) assessed and compared intra-abdominal adhesions using five prosthetic mesh grafts in the repair of abdominal wall defect in rats - PP mesh (Surgipro), PTFE mesh (DualMesh), sodium hyaluronate/carboxymethyl-cellulose-coated polypropylene mesh (Sepramesh), polypropylene/polyglactin 910 composite mesh (Vypro II), resorbable hydrophilic collagen-coated multifiber polyester mesh (PCO mesh). Results showed Parietex caused the least incidence of adhesion formation.

Bellón et al. (2005) tried to modify adhesion formation using different coatings of standard mesh materials in the rabbit model. PP prostheses with physical barriers of expanded PTFE or polyurethane (PU) and prostheses of polyester and PP with absorbable chemical barriers of polyethylene glycol/glycerol and hyaluronate respectively were tested. The observed results showed that only $\mathrm{PP}+\mathrm{PU}$ managed to diminish adhesion formation at the peritoneal interface.

The tissue reaction to mesh material in the human body was analysed in the work of Klosterhalfen et al. (2005) and Klosterhalfen and Klinge (2013). The level of inflammatory infiltrate, connective tissue formation, collagen I/III ratio, some markers of biocompatibility, positive proliferating cells and tunel-positive apoptotic cells in the interface mesh/recipient tissues were reported in the studies of Klosterhalfen and Klinge (2013). Mesh shrinkage, fibrotic bridging, recurrence, chronic pain, fistula, adhesion formation and infection after mesh implantation were also reported using the largest collection of explanted meshes (Klosterhalfen et al., 2005).

Explanted meshes were used to identify physiochemical changes in the surface and bulk properties of the material. Usually, the process is connected with removal of the tissue from the mesh explants. Costello et al. (2007) studied the explanted mesh Composix E/X. The authors reported mesh contraction, loss of compliance and degradation of the material. Wood et al. (2013) described three different synthetic meshes produced by PP, ePTFE and polyethylene terephthalate (PET) explanted from a patient 3 years post-implantation due to ventral hernia recurrence and pain. Scanning electron micrographs and photo micrographs displayed distortion of all three mesh materials.

The long-term performance and changes in the mesh materials however cannot be revealed without mechanical testing of explanted meshes. Our main hypothesis was that long-term changes of mechanical properties of the implanted mesh would influenced the properties of the abdominal wall in the human body. At this stage, we have found no data about the impact of mesh elasticity on rigidity of the abdominal wall. The aim of this study was to analyse the mechanical properties of explanted polyester meshes after their tenure in vivo in order to predict the possible level of rigidity of the abdominal wall following repairs with that type of mesh. 


\section{Materials and methods}

\subsection{Explanted hernia meshes}

The patient was a 60-year-old male who visited the hospital after experiencing severe pain at the hernia site. The patient had a total of three recurrent ventral hernia repairs. The dates of each surgery were February 2009, May 2016 and June 2018. PP (Vypro II) and Parietex meshes were implanted. Parietex meshes were explanted during the second and third recurrent hernia repair surgery in May 2016 and June 2018. The hernia meshes were removed as they had caused infection and pain. The duration period of the last mesh implantation was 2 years.

The study was approved by the Ethics Committee of the Bulgarian Academy of Sciences. Upon removal from the patient, the explanted mesh was placed in bleach at room temperature for 15 days to remove the soft tissue from the mesh. The tests were prepared and it was demonstrated that the cleaning process did not induce any changes in the mechanical properties of the pristine mesh material while the properties of the explanted mesh remained unchanged up to $10 \%$ extension.

The explanted Parietex ${ }^{\mathrm{TM}}$ Composite mesh (Covidien, France) is a three-dimensional double-layer multifilament mesh made from polyester with a collagen hydrogel matrix on the visceral side of the mesh. The hydrophilic and resorbable film is a mixture of oxidized atelocollagen type I, polyethylene glycol and glycerol which protects the viscera from direct contact with the textile (Balique et al., 2005).

The pristine mesh was $150 \mathrm{~mm} \times 100 \mathrm{~mm}$, with $1.5 \mathrm{~mm}-2 \mathrm{~mm}$ of thickness and $78 \mathrm{~g} / \mathrm{m}^{2}$ average weight. The mesh had hexagonal pore shapes with a pore size of $2.4 \mathrm{~mm} \times 2.0 \mathrm{~mm}$. Following the definition of (Amid et al., 1997), this mesh is macroporous with the pore size $>75 \mu \mathrm{m}$.

Figure 1 shows images of the explanted mesh before and after tissue removal.
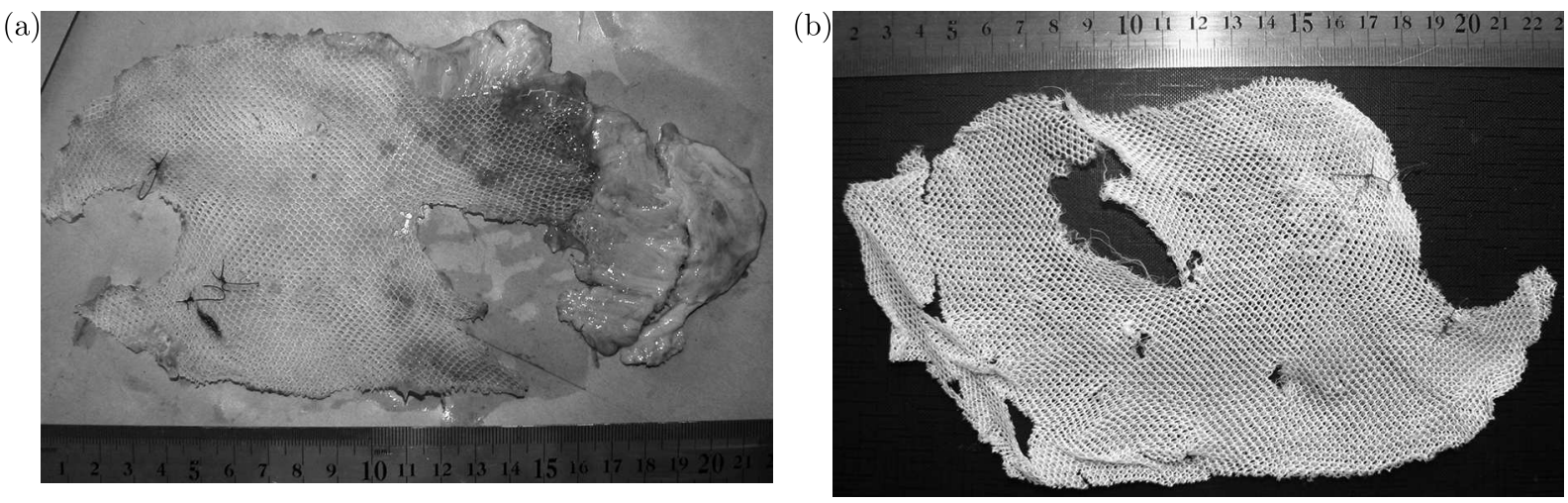

Fig. 1. Photography of the explanted mesh: (a) before tissue removal, (b) after tissue removal

\subsection{Mechanical evaluation}

Two types of mechanical tests were carried out with the explanted mesh: a uni-axial tensile test and a relaxation test. The mesh specimens were cut in two orthogonal directions - longitudinal (along the loop columns; $L$ direction) and transverse (across the loop columns; $T$ direction) to assess their mechanical properties. For each direction, from the pristine material five samples with length of $70 \mathrm{~mm}$ and width of $10 \mathrm{~mm}$ were prepared (a total of 20 samples for both tests). The number of explanted samples was 10 for the tensile test and four for the relaxation test because of the dimension of the explanted mesh. The specimen strips were tested on a universal testing machine equipped with a $500 \mathrm{~N}$ load cell and minimal value of the load of $0.2 \mathrm{~N}$ (Fu1000e, Germany) (Fig. 2). The tests were performed with jaw velocity of the testing machine $0.13 \mathrm{~mm} / \mathrm{s}$ for tensile tests. The initial elongation of samples at $5 \%$ strain during relaxation tests 
was achieved with jaw velocity of $1.26 \mathrm{~mm} / \mathrm{s}$. The mechanical testing was performed at room temperature: as reported in (Dietz et al., 2003), room or body temperatures $\left(37^{\circ} \mathrm{C}\right)$ did not affect the results significantly.

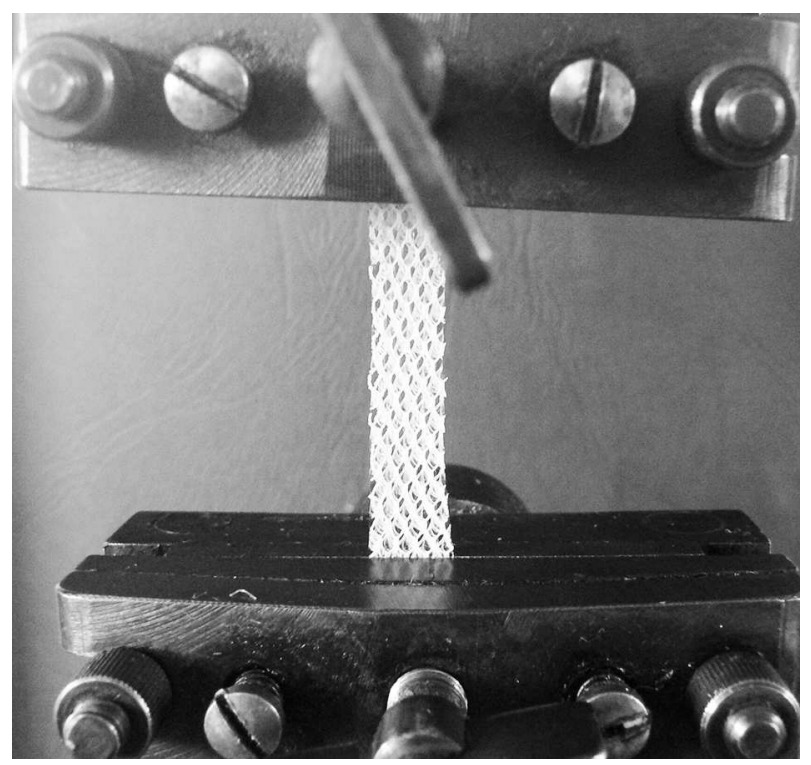

Fig. 2. The specimen in the testing machine

The force and elongation were digitally recorded during the tensile test. Their values were used to calculate Lagrangian stress $T$ and stretch ratio $\lambda$. Lagrangian stress $T$ was calculated as the applied force $F$ divided by the undeformed initial cross-sectional area of the specimen $S$. The stretch ratio $\lambda$ was defined by $\lambda=L / L_{0}$, where $L_{0}$ is the initial length before any load is applied, and $L$ is the sample length after elongation. From the stress-stretch ratio curves, the maximum tensile stress $T_{\text {max }}$, stretch at maximum stress $\lambda_{T_{\max }}$, maximum stretch ratio $\lambda_{\max }$ and secant modulus at $5 \%$ strain $E_{(5)}$ were determined. From the set of five curves obtained in every direction, the average curve was calculated using Origin Software.

The recorded values of the force and time were used to draw the Lagrangian stress-time curves and to present the relaxation. The duration of the relaxation test was $600 \mathrm{~s}$. The initial stress $T_{0}$ at $t=0$, the equilibrium stress $T_{e q}$ at $t=600 \mathrm{~s}$ as well as the modulus at the initial strain $5 \% E_{0}$ and the modulus at the equilibrium state $E_{e q}$ were determined by this test $\left(E_{0}=T_{0} / \varepsilon, \varepsilon=5 \%\right.$, $\left.E_{e q}=T_{e q} / \varepsilon\right)$. The relaxation process was also characterized by reduction of the stress during relaxation. The long-term changes of the relaxation behaviour of the Parietex composite were evaluated using the changes of the stress reduction parameter.

The statistical analysis was performed using MedCalc version 13 Software. The results were presented as the mean \pm standard deviation.

\section{Results}

The pristine and explanted PCO meshes were photographed using a digital camera of light microscope Leica DM 750 with the range of magnification $4 \times-100 \times$ (Fig. 3). The augmentation in both pictures of Figs. $3 \mathrm{a}$ and $3 \mathrm{~b}$ is $5 \times$. The augmented picture of the pristine PCO mesh displayed relatively smooth surfaces while the explanted material showed surface alterations of fibres. The pore size of the meshes was measured using ImageJ Software. The average pore size in the pristine sample was $2.4 \times 2.0 \mathrm{~mm}$ while the explant sample had smaller pores sized $1.79 \mathrm{~mm} \times 1.70 \mathrm{~mm}$. The pore area decreased by $32 \%$. Before implantation, the maximum extension of the samples 
in the orthogonal directions (stretch ratio $\lambda$ ) was 1.6 ( $L$ direction) and 1.45 ( $T$ direction) while after explantation, these values were 1.35 .
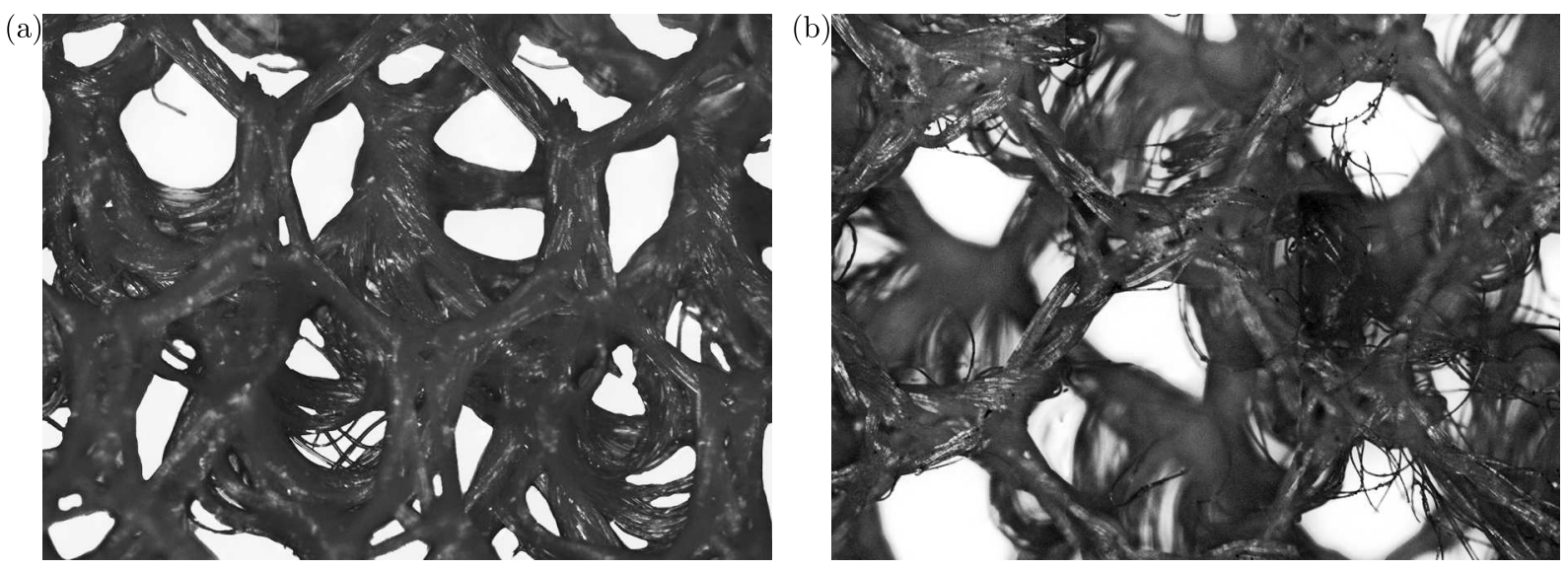

Fig. 3. Augmentation picture of (a) pristine and (b) explanted mesh at $5 \times$

The strength of explanted samples was investigated. The values of $T_{\max }$ decreased from $1.92 \mathrm{MPa}$ to $1.03 \mathrm{MPa}$ in the $L$ direction and increased from $0.82 \mathrm{MPa}$ to $1.04 \mathrm{MPa}$ in the $T$ direction. The elastic modules at $5 \%$ deformation were $3.66 \mathrm{MPa}$ ( $L$ direction) and $1.04 \mathrm{MPa}$ ( $T$ direction), respectively, in preimplantation. After staying in the body for two years, the elastic modulus in the $T$ direction increased 2 times and was $2.11 \mathrm{MPa}$ while the elastic modulus in the $L$ direction remained approximately the same: $3.44 \mathrm{MPa}$ (Fig. 4 and Table 1). The shape of the curves provided information about the process of breaking of the fibres - the multiple peaks denoted this process.

(a)

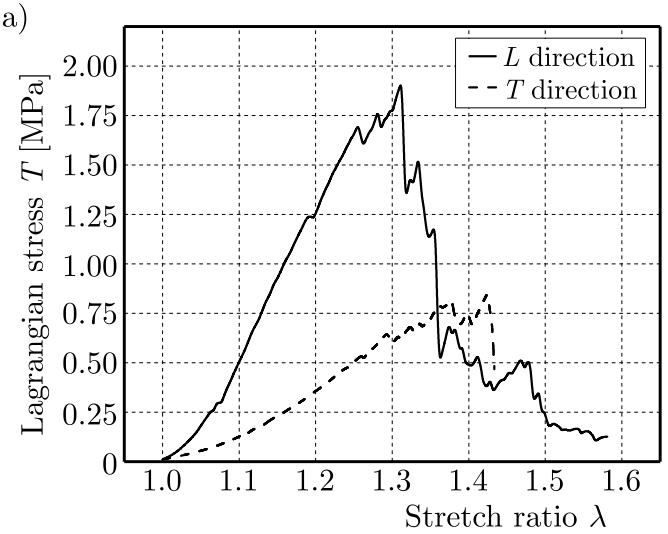

(b)

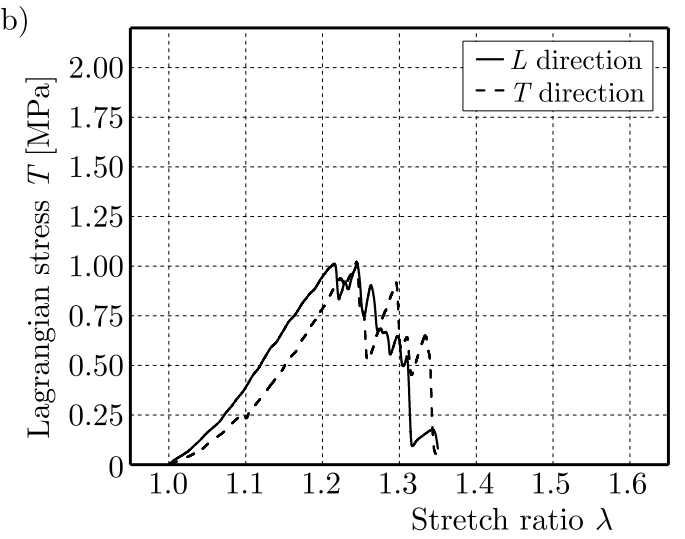

Fig. 4. Average experimental curves for the tensile test: (a) pristine and (b) explanted mesh

Table 1. Mechanical properties of investigated meshes

\begin{tabular}{|l|c|c|c|c|c|}
\hline Mesh group & Direction & $\begin{array}{c}T_{\max } \\
{[\mathrm{MPa}]}\end{array}$ & $\lambda_{T_{\max }}$ & $\begin{array}{c}E_{(5) \lambda_{\max }} \\
{[\mathrm{MPa}]}\end{array}$ & $\lambda_{\max }$ \\
\hline \hline Pristine PCO & $L$ & $1.92 \pm 0.33$ & $1.31 \pm 0.14$ & $3.66 \pm 1.43$ & $1.60 \pm 0.09$ \\
\hline Pristine PCO & $T$ & $0.82 \pm 0.95$ & $1.37 \pm 0.22$ & $1.04 \pm 3.42$ & $1.45 \pm 0.29$ \\
\hline Explanted PCO & $L$ & $1.03 \pm 1.08$ & $1.22 \pm 0.14$ & $3.44 \pm 3.86$ & $1.35 \pm 0.06$ \\
\hline Explanted PCO & $T$ & $1.04 \pm 0.47$ & $1.24 \pm 0.02$ & $2.11 \pm 0.64$ & $1.35 \pm 0.41$ \\
\hline
\end{tabular}


Figure 5 presents the results of relaxation tests of pristine and explanted Parietex mesh samples. The parameters which characterize relaxation - modulus at the initial strain $5 \%-$ $E_{0}$ and modulus at the equilibrium state $E_{e q}$, were calculated. The difference between the mechanical properties of samples in the respective directions was revealed. The mechanical properties of explanted samples showed that $5 \%$ deformation was not sufficient to investigate the relaxation behaviour of the samples. We tested two samples in each direction but the reduction of the stress was very rapid. In the $L$ direction, the total reduction of the stress occurred after an average of 205 seconds ( $73 \mathrm{~s}$ and $335 \mathrm{~s}$ ), and the mean initial stress $T_{0}$ was $0.036 \mathrm{MPa}$ while in the $T$ direction the average value of this parameter was $115 \mathrm{~s}(91 \mathrm{~s}$ and $142 \mathrm{~s}$ ), after the start of relaxation and $T_{0}$ was $0.022 \mathrm{MPa}$. The reduction of stress for the pristine mesh was in the range $33.68 \pm 28.4 \%$ in the $L$ direction and $45.98 \pm 25.17 \%$ in the $T$ direction while for the explanted mesh the range was in the interval 50\%-96.6\% (Fig. 5 and Table 2). Because of the small number of tested explanted samples, we include in Table 2 only parameters from the pristine mesh.
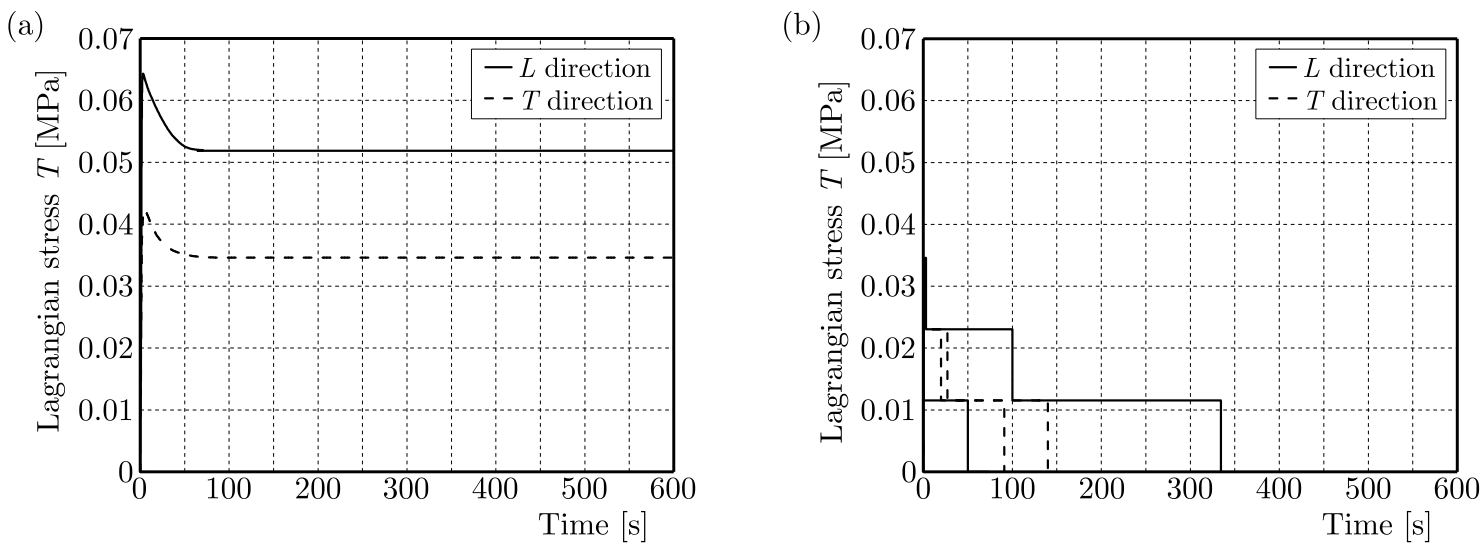

Fig. 5. Experimental results for the relaxation test: (a) pristine (average results) and (b) explanted mesh

Table 2. The main parameters characterizing mechanical properties of the pristine PCO mesh after the relaxation test

\begin{tabular}{|c|c|c|c|c|c|}
\hline Mesh group & Direction & $\begin{array}{c}T_{0} \\
{[\mathrm{MPa}]}\end{array}$ & $\begin{array}{c}T_{e q} \\
{[\mathrm{MPa}]}\end{array}$ & $\begin{array}{c}E_{0} \\
{[\mathrm{MPa}]}\end{array}$ & $\begin{array}{c}E_{e q} \\
{[\mathrm{MPa}]}\end{array}$ \\
\hline \hline Pristine PCO & $L$ & 0.065 & 0.052 & 1.304 & 1.04 \\
\hline Pristine PCO & $T$ & 0.046 & 0.035 & 0.928 & 0.70 \\
\hline
\end{tabular}

\section{Discussion}

The use of composite meshes with coatings of absorbable materials shortens repair duration and reduces risk of complications. A composite hernia mesh of this kind is Parietex, manufactured by Covidien, France, and consisting of non-absorbable polyethylene terephthalate (polyester) with an absorbable collagen coating.

The safety and efficiency of the Parietex Composite mesh (PCO) in the intraperitoneal treatment of incisional and umbilical hernia was reported in (Balique et al., 2005; Chelala et al., 2010; Moreno-Egea et al., 2010). Chelala et al. (2010) reviewed the results of 733 implanted meshes during laparoscopic incisional and ventral hernia repairs. 608 of them were controlled throughout a period of 5 to 10 years. PCO mesh was implanted after redo surgeries. Within the first 3 years, only $4.1 \%$ of the patients experienced recurrences with a mean follow-up of 52 months. The incidence of overall related complications was $18 \%$. The main conclusion of the 
study was that the use of a composite mesh led to a low rate of recurrences and limited side effects.

Parietex is a commercially available mesh in our country. There is information about clinical results reported from Tabakov (2017). The author presented the results from prospective cohort nonrandomized study conducted on 204 patients diagnosed with primary or postoperative ventral hernia. The observation period was from January 2012 to December 2016, the mean age of the patients was $52.30 \pm 12.69$ years in the range of 18 to 87 years. The patients were divided into two groups: group I (operated applying endoscopic surgery) comprising 89 patients $(43.63 \%)$ and group II (applied surgical technique was open surgery) with 115 patients (56.37\%). The operated patients were followed on average 33 months \pm 11.93 months (12-60 months). In all cases, a two-component polyester three-dimensional mesh with collagen anti-adhesive layer Parietex Composite Optimized (CovidienTM) was implanted. The main conclusion was that the laparoscopic approach for the correction of ventral hernias overcame the major drawback of open hernias with a prosthesis - the high incidence of wound infections (10.43\%) - at a similar recurrence rate in the two groups (3.37\% vs. $4.34 \%)$. Obesity remains a major factor in the occurrence of recurrences and hernias at port sites and laparoscopic hernioplasty. In the laparoscopic study group, the overall recurrence rate was significantly higher in patients with Body Mass Index (BMI) $\geqslant 30 \mathrm{~kg} / \mathrm{m}^{2}$ compared to patients with BMI $<30 \mathrm{~kg} / \mathrm{m}^{2}(4.16 \%$ vs. $0 \%)$ (Tabakov, 2017).

According to our previous study which assessed long-term mechanical properties of three pristine heavy-weight hernia meshes with similar density - Surgimesh, Surgipro and Technomesh - the mesh elasticity changed with time but various mesh brands showed different tendencies (Kirilova-Doneva and Pashkouleva, 2019). These meshes were investigated up to four years after their expiration date in our laboratory. The main conclusion from that study was that Technomesh became more elastic, Surgimesh became elastic in one direction only while Surgipro's elasticity showed no significant change. The obtained results attracted our attention and we decided to investigate the mechanical behaviour of explanted hernia meshes.

After implantation, the synthetic mesh had been exposed to factors which altered the mechanical properties of the material and led to its alteration. Wood et al. (2013) described three different synthetic meshes produced by PP, ePTFE and polyethylene terephthalate (PET) explanted from a patient 3 years post-implantation due to ventral hernia recurrence and pain. Scanning electron micrographs and photo micrographs displayed distortion of all three mesh materials. Our results confirmed the alteration of fibres too (Fig. 3).

The shrinkage of Parietex was also assessed, because it is known that mesh shrinkage occurs in a majority of synthetic meshes at a certain level but varies in different types of synthetic mesh materials (Sanbhal et al., 2018). Polyester mesh shrinks from $6.1 \%$ to 33.6\%, PTFE shrinks from $4 \%$ to $51 \%$ and PP from $3.6 \%$ to $25.4 \%$ (Sanbhal et al., 2018). According to Deerenberg et al. (2016), the bulging at the hernia repair site of implanted Parietex was caused by elongations of the mesh due to pore enlargement. Our results showed a $20 \%$ decreased mesh elongation in the $L$ direction and decreased area of investigated mesh pores by $32 \%$. The decreased elongation of the samples and dimension of pore sizes probably showed shrinkage of the mesh samples. More samples are necessary in order to be sure that the results confirm the mesh shrinkage.

The obtained results for the PCO mesh indicates that the tendencies of the main tensile properties of Parietex are completely different from those presented in (Kirilova-Doneva and Pashkouleva, 2019) for Surgimesh, Surgipro and Technomesh. Decrease of strength and increase of stiffness of the PCO mesh in one direction was observed. The initial values of the maximum stress $T_{\max }$, which is in the range $0.82-1.92 \mathrm{MPa}$, were less than those previously reported, which were in the interval 8-11 MPa. The values of the maximum stretch $\lambda_{\max }$ were between 1.35-1.6 and did not exceed 1.7. The secant modulus $E_{(5)}$ of Surgimesh, Surgipro and Technomesh was between 4.36-12.63 $\mathrm{MPa}$ while the secant modulus $E_{(5)}$ of Parietex mesh samples was between 
1.07-3.98 MPa. Results indicated that Technomesh was the strongest mesh, at about five times the strength of Parietex. Parietex was the least extensible mesh which was able to increase by only $45-60 \%$ as opposed to Technomesh which was able to double its original length. The meshes demonstrated linear loading curves with a relatively high stiffness. The impact of time on the elasticity of Parietex was demonstrated in the $T$ direction (Fig. 4). The secant modulus $E_{(5)}$ for Parietex increased by about two times in the $T$ direction, but in the $L$ direction the modulus of Parietex remained constant. Parietex did not show preserved mechanical stability over a period of two years as Surgipro did. Thus Parietex has completely unique mechanical behaviour - it is less strong and less stiff.

Lubowiecka et al. (2016) considered Parietex as a distinctly bilinear elastic orthotropic material with different orthotropy ratios calculated as $E_{1} / E_{2}$, where $E_{1}$ and $E_{2}$ are the elastic moduli of the mesh derived in two perpendicular directions $\left(E_{1}>E_{2}\right)$. They investigated material behaviour in the strain range up to $30 \%$. According to our results, the PCO mesh material is also bilinear but the strain at which the material shows this property depends on the direction of elongation. The pristine material changes its elastic modulus after $2.3 \%$ ( $L$ direction) and $10 \%$ ( $T$ direction). Explanted samples exhibit their bilinearity after $7.1 \%$ and $5.1 \%$ accordingly. The aim of our study was to characterize qualitatively the main mechanical properties of explanted polyester meshes, which was the reason to use one elastic modulus for every direction.

Hernia meshes can provide the strength needed in hernia repair. According to recent investigations. normal daily activities require a tensile strength of $16 \mathrm{~N} / \mathrm{cm}$, and strenuous activities require a maximum tensile strength of $42-47 \mathrm{~N} / \mathrm{cm}$ (Deeken et al., 2011; Klinge et al., 1998). It was reported in (Doneva and Pashkouleva, 2017) that the load transferred to Surgimesh in both directions should be no more than $32 \mathrm{~N} / \mathrm{cm}$ while Surgipro and Technomesh can withstand load $47 \mathrm{~N} / \mathrm{cm}$ in the $L$ and $T$ direction. For Parietex, we observed that the maximum applied load for pristine mesh was $36.2 \pm 6.2 \mathrm{~N} / \mathrm{cm}$ in the $L$ direction and $20.11 \pm 1.09 \mathrm{~N} / \mathrm{cm}$ in the $T$ direction. The maximum value of the applied load for the explanted mesh was $19.54 \pm 2.96 \mathrm{~N} / \mathrm{cm}$ and $19.48 \pm 3.05 \mathrm{~N} / \mathrm{cm}$, respectively.

The deformation at $16 \mathrm{~N} / \mathrm{cm}$ for the previously studied meshes Surgimesh, Surgipro and Technomesh, was $42-78 \%$ in the $L$ direction and $15-48 \%$ in the $T$ direction (Doneva and Pashkouleva, 2017). The results for pristine Parietex were $16.44 \pm 3.8 \%$ in the $L$ direction and $44.6 \pm 13.35 \%$ in the $T$ direction. For the explanted mesh, the deformation at $16 \mathrm{~N} / \mathrm{cm}$ was $28.22 \pm 12.9 \%$ in the $L$ direction and $26.05 \pm 2.52 \%$ in the $T$ direction. Thus the difference of the maximum applied load between both directions of $28 \%$ before implantation decreases to $2 \%$ after implantation.

The results of relaxation behaviour showed that initial stress for Parietex is less than the stress calculated for other heavy weight meshes $(0.524-0.145 \mathrm{MPa})$. The value of $T_{0}$ for Parietex is $0.065 \mathrm{MPa}$ in the longitudinal direction and $0.045 \mathrm{MPa}$ in the transversal direction. The stress reduction is close to that previously reported for Surgimesh, Surgipro and Technomesh.

Some limitations in the study can be mentioned. The results about the decreased pore size correspond with the studies of Deerenberg et al. (2016) and Sanbhal et al. (2018) analysing surface deformation of the meshes. More experiments, however, are necessary to justify the mesh shrinkage. The effect of a bleach on the alteration of mesh fibres was not considered. Usually the bleach is used to remove the tissue remnants (Costello et al., 2007; Wood et al., 2013). We have experienced with polypropylene (PP) and polyester (PE) meshes soaked in a bleach, but only the mechanical properties of these meshes were investigated. The results for PP were published in (Kirilova-Doneva et al., 2015). As the mechanical properties of the PE mesh before and after bleach bath were close we concluded that the bleach does not influence mesh fibres. Actually, the impact of the bleach on mesh fibres should be observed in new experiments.

Perspectives of this study are to continue mechanical testing with different mesh explants. 


\section{Conclusion}

Implantation of synthetic meshes provokes complications which require long-lasting follow-up. The synthetic mesh materials exposed to physiological conditions demonstrate differences in failure load, stiffness and extension.

Mechanical alterations of a polyester mesh of human explants were presented. We found a difference in the biomechanical properties when comparing Parietex meshes both pre-implantation and at two years post-implantation. The tensile strength of explanted samples was changed in the transversal direction. The elastic modulus in the same direction increased two times. The relaxation tests revealed that the initial stress decreases after implantation, whereas the reduction of stress increases. Further tests at physiological loads are necessary in achieving relevant biomechanical data about explanted meshes.

\section{References}

1. Amid P., 1997, Classification of biomaterials and their related complications in abdominal wall surgery, Hernia, 1, 1, 15-21

2. Balique J.G., Benchetrit S., Boulllot J.L., Flament J.B., Goulllat C., Jarsaillon P., Lepère M., Mantion G., Arnaud J.P., Magne E., Brunetti F., 2005, Intraperitoneal treatment of incisional and umbilical hernias using an innovative composite mesh: four-year results of a prospective multicenter clinical trial, Hernia, 9, 1, 68-74

3. Bellón J.M., Serrano N., Rodriguez M., Garcia-Honduvilla N., Pascual G., Buján J., 2005, Composite prostheses used to repair abdominal wall defects: physical or chemical adhesion barriers?, Journal of Biomedical Materials Research Part B, 74, 2, 718-724

4. Chelala E., Debardemaeker Y., Elias B., Charara F., Dessily M., Allé J.L., 2010, Eighty-five redo surgeries after 733 laparoscopic treatments for ventral and incisional hernia: adhesion and recurrence analysis, Hernia, 14, 2, 123-129

5. Costello C.R., Bachman S.L., Ramshaw B.J., Grant S.A., 2007, Materials characterization of explanted polypropylene hernia meshes, Journal Biomedical Materials Research Part B, 83, 1, 44-49

6. Deeken C.R., Abdo M.S., Frisella M.M., Matthews B.D., 2011, Physicomechanical evaluation of polypropylene, polyester, and polytetrafluoroethylene meshes for inguinal hernia repair, Journal of the American College of Surgeons, 212, 1, 68-79

7. Deerenberg E.B., Verhelst J., Hovius S.E.R., Lange J.F., 2016, Mesh expansion as the cause of bulging after abdominal wall hernia repair, International Journal of Surgery Case Reports, 28, 200-203

8. Dietz H.P., Vancaillie P., Svehla M., Walsh W., Steensma A.B., Vancaillie T.G., 2003, Mechanical properties of urogynecologic implant materials, International Urogynecol Journal, 14, 4, 239-243

9. Doneva M., Pashkouleva D., 2017, Practical recommendations for application of hernia meshes, Series on Biomechanics, 31, 2, 34-40

10. Hawn M.T., Gray S.H., Snyder C.W., Graham L.A., Finan K.R., Vick C.C., 2011, Predictors of mesh explantation after incisional hernia repair, American Journal of Surgery, 202, 1, $28-33$

11. Jacob B.P., Hogle N.J., Durak E., Kim T., Fowler D.L., 2007, Tissue ingrowth and bowel adhesion formation in an animal study: polypropylene vs. Proceed vs. Parietex Composite, Surgical Endoscopy, 21, 4, 629-633

12. Judge T.W., Parker D.M., Dinsmore R.C., 2007, Abdominal wall hernia repair: a comparison of Sepramesh and Parietex Composite mesh in a rabbit hernia model, Journal of the American College of Surgeons, 204, 2, 276-281 
13. Kayaoglu H.A., Ozkan N., Hazinedaroglu S.M., Ersoy O.F., Erkek A.B., Koseoglu R.D., 2005, Comparison of adhesive properties of five different prosthetic materials used in hernioplasty, Journal of Investigative Surgery, 18, 2, 89-95

14. Kirilova-Doneva M., Pashkouleva D., 2019, Long-term mechanical compatibility of polypropylene surgical meshes, Journal of Mechanics in Medicine and Biology, 19, 6, 1950056

15. Kirilova-Doneva M., Pashkouleva D., Kavardzhikov V., Sopotensky S., Petrova G., Gerasimov N., 2015, Evaluation of mechanical alterations of explanted hernia mesh, Series on Biomechanics, 29, 2-3, 73-77

16. Klinge U., Klosterhalfen B., Conze J., Limberg W., Obolenski B., Ottinger A.P., Schumpelick V., 1998, Modified mesh for hernia repair that is adapted to the physiology of the abdominal wall, The European Journal of Surgery, 164, 12, 951-960

17. Klosterhalfen B., Junge K, Klinge U., 2005, The lightweight and large porous mesh concept for hernia repair, Expert Review of Medical Devices, 2, 1, 103-117

18. Klosterhalfen B., Klinge U., 2013, Retrieval study at 623 human mesh explants made of polypropylene impact of mesh class and indication for mesh removal on tissue reaction, Journal of Biomedical Materials Research Part B, 101, 8, 1393-1399

19. Lubowiecka I., Szepietowska K., Szymczak C., Tomaszewska A., 2016, A preliminary study on the optimal choice of an implant and its orientation in ventral hernia repair, Journal of Theoretical and Applied Mechanics, 54, 2, 411-421

20. McGinty J.J., Hogle N.J., McCarthy H., Fowler D.L., 2005, A comparative study of adhesion formation and abdominal wall ingrowth after laparoscopic ventral hernia repair in a porcine model using multiple types of mesh, Surgical Endoscopy, 19, 6, 786-790

21. Moreno-Egea A., Bustos J.A., Girela E., Aguayo-Albasini J.L., 2010, Long-term results of laparoscopic repair of incisional hernias using an intraperitoneal composite mesh, Surgical Endoscopy, 24, 2, 359-365

22. Sanbhal N., Miao L., Xu R., Khatri A., Wang L., 2018, Physical structure and mechanical properties of knitted hernia mesh materials, Journal of Industrial Textiles, 19, 6, 1266-1270

23. Song Ch., Alijani A., Frank T., Hanna G., Cuschieri A., 2006, Elasticity of the living abdominal wall in laparoscopic surgery, Journal of Biomechanics, 39, 587-591

24. TABAKov M., 2017, Research on opportunities for treatment of ventral hernias with endoscopic techniques, Ph.D. Thesis, Medical University - Sofia

25. Tran D., Podwojewski F., Beillas P., Ottenio M., Voirin D., Turquier F., Mitton D., 2016, Abdominal wall muscle elasticity and abdomen local stiffness on healthy volunteers during various physiological activities, Journal of Mechanical Behavior of Biomedical Materials, 60, $451-459$

26. Wood A.J., Cozad M.J., Grant D.A., Ostdiek A.M., Bachman S.L., Grant S.A., 2013, Materials characterization and histological analysis of explanted polypropylene, PTFE, and PET hernia meshes from an individual patient, Journal of Materials Science: Materials in Medicine, 24, 4, 1113-1122 